\title{
Complementary Pharmacological Treatment and Therapeutic Prospects for COVID-19
}

\author{
Daphne Foust ${ }^{1}$, Shazia Tabassum Hakim ${ }^{2}$ and Joseph Angel de Soto ${ }^{2 *}$ \\ ${ }^{1}$ Foust Medical Clinic, Paris, France \\ ${ }^{2}$ School of Science, Technology, Engineering and Math, Dine College, Arizona, USA \\ *Corresponding Author: Joseph Angel de Soto, School of Science, Technology, \\ Engineering and Math, Dine College, Arizona, USA.
}

Received: September 07, 2020

Published: October 28, 2020

(C) All rights are reserved by Joseph Angel de

Soto., et al.

\begin{abstract}
Background: The COVID-19 outbreak has led to a world-wide pandemic that has infected over two million people and caused over one hundred thousand deaths. The virus first discovered in Wuhan China in Dec. 2019, seems to have originated either in the United States or the Guangdong province as early as September 2019. Infection by SARs-CoV-2 virus can lead to pneumonia and adult respiratory distress syndrome (ARDS) which is the leading cause of morbidity and mortality for this disease.

Objective: This review focuses on the mechanism of action of a representative sampling of some of the most promising pharmacological approaches to treating COVID-19 based pneumonia and the related cytokine storm.

Results: The current medications showing promise either focus in interfering with the viral life cycle or interfering with the cytokine storm that leads from pneumonia to ARDs.

Conclusion: Developing therapeutics interfering with the viral lifecycle, modulating the cytokine storm and protecting the end organs show promise in significantly reducing the morbidity and mortality of COVID-19.
\end{abstract}

Keywords: Covid-19; Therapeutics; Antiviral; Cytokine Storm; Therapy; Treatment; Pharmacological; SAR-CoV-2

\section{Background}

The SARs-CoV-2 virus was first discovered in the city of Wuhan in December of 2019 following a pneumonia outbreak. Growing evidence suggests that the virus may have been brought to the city of Wuhan having originated either in the Guangdong or the United States as early as September of 2019 according to phylogenetic analysis [1]. As of 18 April 2020, there have been 2,310,572 confirmed cases and 158,691 deaths. Perhaps, what separates COVID-19 from SARs and MERs diseases is its transmissibility. Currently there are over 300 subtypes and perhaps three major strands of this virus [2]. On average a person will transmit the virus to 3.28 individuals [3]. This in part is due to the ability of the virus to be transmitted when a person is asymptomatic who often has the same viral load as an infected person [4,5]. It is reported that an infected person can shed virus up to 34 days and remain viable for up to 72 hours as a fomite [6,7].

The average time of infection to symptoms is about 5.8 days with the time to hospitalization being 12.5 days [8]. The most common presentation for infection for COVID-19 is fever $88.7 \%$, cough $67.8 \%$, fatigue $38.1 \%$, productive sputum $33.7 \%$ and shortness of breath $18.7 \%$ with shortness of breath being the most ominous [9]. Among the most important comorbidities for severe disease are diabetes, hypertension and cardiovascular disease with prior respiratory disease and obesity not as significant [10]. Those with diabetes often have an impaired immune system and are much more likely to obtain not just COVID-19 based pneumonia but pneumonia in general $[11,12]$. Increased insulin resistance occurs 
during infection which causes metabolic instability [13]. In addition, the SARs CoV-2 virus may directly damage the islets of Langerhans [14]. Those with hypertension and cardiovascular disease face increasing pulmonary hypertension and inflammation of the vascular system which can lead to arrhythmias, cardiac failure and cardiac arrest. Though those who are obese tend to shed virus for a longer time than those who are not obese, obesity seems to be a protective factor for viral pneumonia when isolated from its confounders of diabetes and hypertension [15-17].

Up to $99.3 \%$ of those admitted to the hospital have pneumonia with $89.6 \%$ having involvement of both lobes. Among those that do not survive, 93\% have ARDS, 98\% respiratory failure and near $100 \%$ sepsis. The high mortality rate and progression to ARDs and subsequent respiratory failure and sepsis in COVID-19 seems to be in part related to an excessive immune response called a cytokine storm [18]. The cytokine storm involves a massive activation of Tcells, B-cells, endothelial cells, macrophages, dendritic cells, monocytes, neutrophils, and natural killer cells. The following cytokines have been identified as part of the cytokine storm: IL-1, IL-6, IL-12, IL-16, IL-17, IL-18, TNF- $\alpha$, INF- $\gamma$, and INF-a2. High elevations of IL-6 have especially been correlated with a higher mortality rate with IL-17 amplifying the response to IL- 6 by increasing IL- 6 levels. This storm leads to ARDs, lung fibrosis, hypotension, bleeding secondary to liver failure, and finally renal failure [19]. The cytokine storm may be more important in overall mortality than viral load itself [20].

\section{SARs-Cov-2 virus life cycle}

The SARs-CoV-2 virus begins its lifecycle by using it $\mathrm{S}$ surface protein to bind to the ACE2 receptor of the cell. The receptor is found in large numbers in the oral and nasal mucosa, type II pneumocytes of the lung, blood vessels, intestines, spleen, and liver [21]. A mutation in the $\mathrm{S}$ surface protein allows this protein to bind with a higher affinity to the ACE2 receptor than the previous SARS and MERS corona viruses [22]. After binding to the receptor, the S protein changes its conformation which allows the viral phospholipid membrane to fuse with the cellular phospholipid membrane allowing for the virus to enter the cell via the endosomal pathway. Once inside the endosome the virus is uncoated freeing its genomic + strand of RNA. This viral RNA is transcribed discontinuously creating both genomic and non-genomic RNA. Cellular polymerase then translates sub-genomic RNA into viral proteins at the endoplasmic reticulum. The viral proteins assemble around the genom- ic RNA and are packed at the Golgi Apparatus for exocytosis of the newly created virus outside of the cell. The life cycle, elucidates the following pharmacological targets for intervention 1) binding of the S protein with the ACE2 receptor, 2) uncoating of the virus, 3) transcription of the viral RNA, 4) translation of the viral RNA, 5) assembly of the virus and 6) the exocytosis of the virus. Yet, these targets may represent a moving target as there have been reported to be over 300 variants of the SARs-CoV-2 virus with variance of variance ranging between 270 fold from the least to most virulent [23].

\section{Anti-virals}

Azithromycin an antibiotic may not only reduce the risk of secondary bacterial infection due to COVID-19 and has been shown to have anti-viral properties and limit inflammation in part by reducing IL-6 and IL-8 [24]. Azithromycin has been shown to induce INF- $\beta$ and INF- $\gamma$ in patients with a compromised pulmonary system $[25,26]$. INF is essential in fighting the early stages of SARsCoV-6 infection [18], In addition, INF inhibit the invasion of the pulmonary system by neutrophils [27]. Azithromycin inhibits the bronchial excretion of mucin which when excreted narrows the bronchial passageways inhibiting breathing thus, Azithromycin may functionally improve breathing. INF- $\beta$ and INF- $\gamma$ are elevated in the cytokine storm but, the elevation seems to be in proportion to viral load.

Chloroquine has been used since 1934 as an antimalarial and immunosuppressant. Chloroquine has been shown to form an ionophore in the cellular membrane allowing doubling the amount of zinc another anti-viral that can enter the cell at clinically relevant levels [28]. Chloroquine is a very alkaline medication that can readily distribute throughout one's cells and settle in those areas that are especially acidic such as the endosomes which are small vacuoles that the COVID -19 virus uses to enter and uncoat. Chloroquine can increase the $\mathrm{pH}$ of the endosome inhibiting the ability of the COVID-19 virus to enter the cell. Additionally, the changing of the $\mathrm{pH}$ of the endoplasmic reticulum and Golgi apparatus inhibit the ability of the virus to assemble and be released from the cell [29]. In a clinical trial of 100 patients using chloroquine it was found that the course of the disease was shortened, and the pneumonia exasperation inhibited without any serious side effects [30]. Hydroxychloroquine may have greater inhibitory potential than chloroquine and does not have the same risk to cause retinal damage [31]. Yet, like Chloroquine, Hydroxychloroquine has a narrow therapeutic index and must be dosed appropriately [32]. Hydroxychlo- 
roquine levels may be 400 times higher in the lung and other tissues than in the in the blood. Inappropriate dosing of chloroquine may account for the early termination of the Brazilian study that was stopped prematurely due cardiac arrhythmia. In that study the low dose was $450 \mathrm{mg}$ day B.I.D while the high dose was $600 \mathrm{mg}$ B.I.D. with the so-called low dose being more than twice as high as the standard dosing of $200 \mathrm{mg}$ day B.I.D. [32,33].

Favipiravir is a drug used in Japan for influenza. Favipiravir is a prodrug that is metabolized to the active form favipiravir-ribofuranosyl-5'-triphosphate (favipiravir-RTP) [34]. Favipiravir-RTP competes with purine nucleosides for incorporation into the viral RNA and once incorporated interferes with RNA polymerase causing chain termination [35]. In a small study, 80 patients with COVID were placed either in a Favipiravir arm or an arm with lopinavir and ritonavir. The patients in the Favipiravir arm did significantly better with $22.9 \%$ improving after 4 days of treatment, $25.7 \%$ worsening and $51.4 \%$ staying the same. After 14 days a treatment this changed to $91.4 \%$ improving, $3.2 \%$ worsening and $6.45 \%$ remaining the same [36]. The adverse effects were $5.7 \%$ with diarrhea and $2.86 \%$ with elevated liver enzymes. It has been shown that i viral models were resistance develops due to poor incorporation of Favipiravir into viral RNA that these viruses grow much slower than non-resistant strains [37].

Ivermectin since 1981 has been listed as one of the World's essential medications. This medication is generally used for worm and other parasitic infections such as scabies. This drug is a macrocyclic lactone with a related structure to the macrolides. Ivermectin appears to inhibit helicase in viruses [38]. Ivermectin in vitro inhibits SAR-CoV-2 replication $99.98 \%$ at $5 \mu \mathrm{M}$ in vitro. However, 5 $\mu \mathrm{M}$ is a much higher level than reached in vivo [39]. It remains to be seen how effective ivermectin will be in vivo at lower levels against SAR-CoV-2. Among the side effects of Ivermectin are reduced factor II and factor VII with prolonged prothrombin times [39].

Remdesivir is a prodrug that must be metabolized into its active form GS-441524 which is an adenosine nucleotide analog [40]. GS-441524 interferes with the action of viral RNA-dependent RNA polymerase and can evade proofreading by viral exoribonuclease. This causes a decrease in viral RNA production. In a cohort study without a control $68 \%$ of patients with severe COVID-19 treated with Remdesivir showed clinical improvement [41]. A recent randomized control study of 158 patients showed no statistical difference in mortality rate with a slower rate of improvement shown by patients in the Remdesivir arm. The patients however were allowed to enter the trial up to 15 days after COVID-19 disease appeared [42]. The differences in trial outcomes might be due to different strains in SARs-CoV-2. More trials should clarify the picture.

Umifenovir has been used for 30 years to combat seasonal influenza and is used widely today to treat RNA viruses inclusive of: $\mathrm{Or}$ thomyxoviridae, Paramyxoviridae, Bunyaviridae, Rhabdoviridae, Togaviridae, Hepadnaviridae, Hepaciviridae, Filoviridae, Flaviviridae and Arenaviridae [43], The mechanism of action for Umifenovir is to block the formation of the endosome ad other membrane bound organelles thereby blocking viral uncoating the release of the genome [43]. There is also evidence that Umifenovir can protect the mitochondrial production of ATP and hence, limit cellular death of cells. This would be important in limiting the death of Type II pneumocytes and maintain the ability to produce adequate amounts of surfactant [44]. In a clinical trial in China sixteen COVID-19 patients with fourteen having pneumonia were administered Umifenovir $200 \mathrm{mg}$ T.I.D while hospitalized. All sixteen patients survived, and no one developed ARDs [45], [46]. Among the side effects in this study $68.7 \%$ of patients had elevated bilirubin and $43.7 \%$ patients demonstrated digestive upsets. None withdrew due to toxicity [46]. Imatinib is another drug with a similar mechanism of action to Umifenovir [47].

Zinc has been shown to limit the duration of colds (many of which are caused by coronaviruses) by $40 \%$ in duration [48]. Zinc as a cofactor for may enzymes involved in both the innate and adaptive immune system is necessary in adequate amounts for phagocytosis of infected cells, killing of infected cells by the NK cells and the blocking of the entrance of viruses into the cells by opsonization [49]. Zinc also may also interfere with RNA polymerase the necessary protein essential for the replication genomic RNA [50]. Zinc may be most useful for those who have a Zinc deficiency and may possibly increase the threshold for infection with SARs-CoVSARs2.

\section{Cytokine storm inhibitors}

Anakinra is an interleukin 1 receptor antagonist and has been shown to be useful in macrophage activation syndrome (MAS), a form of cytokine storm [51]. Interleukin 1 is a family of 11 cytokines with interleukin $1 \alpha$ and interleukin $1 \beta$ both binding to the interleukin 1 receptor. Both interleukin $1 \alpha$ and $1 \beta$ are produced early during SAR-CoV-2 infection by macrophages and dendritic cells, causing fever, vasodilation, and hypotension as part of the innate 
immune system [52]. In a small study of 5 severely ill patients with cytokine storm of the macrophage subtype received continuous infusion of up to $2400 \mathrm{mg} /$ day. Four of the five patients responded with three of the five recovering completely [53]. The most common side effect of Ankinra is a rash [54].

Baricitinib is a competitive adenosine triphosphate kinase inhibitor that prevents the transfer of a phosphate group to Janus Kinase 1 and Janus Kinase 2 (JAK1, JAK2) the activators of these two kinases. JAKs play a role in the signal transduction of cytokines to signal transducers and activators of transcription (STAT) pathways. Baricitinib by inhibiting the activation of the JAK1 and JAK2 kinases inhibits the signal transduction of erythropoietin, IL-2, IL6, IL-23, G-CSF, GM-CSF, INF- $\alpha$, INF- $\beta$, INF- $\gamma$, and other cytokines $[55,56]$. Among the side effects of Baricitinib are a small increase in pulmonary embolisms or DVT's (0.5 - 1.0\%), a small decrease in reticulocytes, a transient increase in hepatic transaminases [57]. Baricitinib reduces systemic inflammation in disease such as lupus, rheumatoid arthritis and also has protective effect on the kidney [56]. Baricitinib holds promise for COVID-19.

Tocilizumab is a recombinant humanized antibody of the IgG1 subclass that blocks the binding of IL- 6 with the interleukin - 6 receptor [58]. Interleukin- 6 inhibits IL-1, TNF- $\alpha$ and regulatory T-cells while activating B-cells, macrophages, neutrophils and induces the differentiation of Helper T cells [59]. Interleukin-6 also elevates C-reactive protein which is a general marker for inflammation. Obesity and exercise both increase interleukin-6 levels $[60,61]$. Since, IL-6 plays a central role in the pathogenesis of cytokine storm the blockage of it may play a significant role in the amelioration of the cytokine storm which in turn may limit morbidity and mortality. The most common immediate side effects are headache $1 \%$, skin reactions $1 \%$, and hypersensitivity reactions causing discontinuation $0.2 \%$ [58]. Tocilizumab may decrease levels of statins and cyclosporin due to elevation of the cytochrome system [61]. Alternatively, tocilizumab may increase levels of theophylline, warfarin, phenytoin, and phenprocoumon [59]. Sarilumab is another inhibitor of interleukin -6 receptor it is administered subcutaneously while Tocilizumab is administered through IV. There is no difference between the two in terms of adverse effects [62].

Infliximab is a chimeric human murine monoclonal antibody that binds to TNF- $\alpha$. TNF- $\alpha$ is produced chiefly by macrophages, though CD4+ lymphocytes, eosinophils, mast cells, NK cells, neurons and neutrophils. TNF- $\alpha$ is a chemoattractant for neutrophils, induces insulin resistance plays a role in shock and limits viral production. Hence, the blocking of TNF- $\alpha$ by infliximab may be useful in blocking cytokine storm especially in diabetics. The blocking of TNF- $\alpha$ also down regulates Il- 6 which seems central in the cytokine storm [63]. Some of the side effects of Infliximab are infusion reactions, $10.74 \%$, pain $7.05 \%$, abnormal liver tests $2.92 \%$ and headache $2.64 \%$ [64]. Long term use of infliximab may lead to increased susceptibility to tuberculosis, hepatitis and influenza and a decreased response to vaccinations [65]. Adalimumab is another TNF blocker but unlike infliximab is a completely human antibody and is similar to infliximab [66]. Since TNF- $\alpha$ inhibits viral replication and argument can be made that TNF- $\alpha$ blockers might best be used later in the disease process specifically to limit the cytokine storm. As in the case of Anakinra, Infliximab seems to be more effective when given continuously as opposed to intermittently [20].

\section{Supportive adjuvants}

Melatonin is a hormone that regulates the sleep-wake cycle and is secreted by the pineal gland. This hormone also has anti-inflammatory properties mediated by the up-regulation of Sirtuin. Up regulation of Sirtuin may have a modest protective effect toward the lung [29]. Daily use of melatonin also reduces levels of TNF- $\alpha$, IL-6, and IL-1 $\beta$. Lastly, in a meta-analysis melatonin improves the sleep quality of patients in ICU increasing survival rates $[67,68]$. Among the side effects of Melatonin are abnormal dreams, headaches, nausea, and diarrhea [69].

Steroids are a useful tool in reducing inflammation and improving breathing in many pulmonary diseases including asthma and some types of pneumonia. In SARs-CoV-2 pneumonia the lung undergoes massive bilateral inflammation [70]. Yet, early evidence and experience with SARs-CoV and MERS suggests that steroids may prolong the shedding of virus and increase mortality [71,72]. Steroids cannot be recommended at this time [73].

Bevacizumab is a recombinant humanized monoclonal antibody that blocks angiogenesis by inhibiting vascular endothelial growth factor A (VEGF-A). VEGF is associated with leaky capillaries, interstitial thickening, pulmonary edema and permanent remodeling of the lung which not only threaten mortality but if the patient survives leave the patient with permanently reduced lung function [74]. Bevacizumab has been shown to reduce these features in ARDs and is often used in these patients in ICU $[75,76]$. A concern with the use of Bevacizumab is hypertension, worsen- 
ing coronary, and peripheral heart disease [77]. This drug may be useful in ameliorating ARDs and inhibiting the restructuring of the lung from COVID-19.

\section{Conclusion}

Improved pharmacological treatment is needed to improve the morbidity and mortality of those with COVID-19. Once place on mechanical ventilation for COVID-19 provides the survival rate may be as low as $5.5 \%$ [78]. The poor mortality and morbidity observed with COVID-19 appears to be due to the cytokine storm [79]. There seems to be little relationship between the early stages of the disease and predicting who does or does not develop severe disease [18]. In sum, the evidence suggests that the viral inhibitors may be more efficient early in the disease and not as effective once the progression to ARDs has begun, here is where the cytokine storm inhibitors may play the greatest role in limiting mortality. Additionally, the experience gained with SARs-CoV and MERS suggests that the tri-fold approach of limiting viral dissemination, inhibiting the cytokine storm and protecting the lungs and other organs through specific targeting will provide the best outcome [80].

\section{Acknowledgements}

Bonnie Grunther for reviewing the manuscript.

\section{Conflict of Interests}

None.

\section{Bibliography}

1. Forster P., et al. "Phylogenetic Network Analysis of SARS-CoV-2 Genomes". Proceedings of the National Academy of Sciences of the United States of America 117.17 (2020): 9241-9243.

2. Ceraolo C and Giorgi FM. "Genomic Variance of the 2019-nCoV Coronavirus". Journal of Medical Virology (2020).

3. Liu Y., et al. "The Reproductive Number of COVID-19 is Higher Compared to SARs Coronavirus". Journal of Travel Medicine 27.2 (2020).

4. Rothe C., et al. "Transmission of 2019-nCoV Infection from an Asymptomatic Contact in Germany". New England Journal of Medicine 382 (2020): 970-971.

5. Zou L., et al. "SARs-CoV-2 Viral Load in Upper Respiratory Specimens of Infected Patients". New England Journal of Medicine 382 (2020): 1177-1179.
6. Zhou F., et al. "Clinical Course and Risk Factors for Mortality of Adult Inpatients with COVID-19 in Wuhan, China: A Retrospective Cohort Study". Lancet 395 (2020): 1054-1062.

7. van Doremalen N., et al. "Aerosol and Surface Stability of SARSCoV-2 as Compared with SARS-CoV-1". New England Journal of Medicine 382 (2020): 1564-1567.

8. Li Q., et al. "Early Transmission Dynamics in Wuhan, China of Novel Coronavirus - Infected Pneumonia". New England Journal of Medicine (2020).

9. Guan W., et al. "Clinical Characteristics of Coronavirus Disease 2019 in China". New England Journal of Medicine 382 (2020): 1708-1720.

10. Yang J., et al. "Prevalence of Comorbidities in the Novel Wuhan Coronavirus (COVID-19) Infection: A Systemic Review and Meta-Analysis". International Journal of Infectious Diseases 94 (2020): 91-95.

11. Jensen AV., et al. "Undiagnosed Diabetes Mellitus in Community Acquired Pneumonia: A Prospective Cohort Study”. Clinical Infectious Disease 65.12 (2017): 2091-2098.

12. Fadnini GP., et al. "Prevalence and Impact of Diabetes Among People Infected with SARS-CoV-2". Journal of Endocrinological Investigation (2020).

13. Yki-Järvinen H., et al. "Severity, duration, and mechanisms of insulin resistance during acute infections". The Journal of Clinical Endocrinology and Metabolism 69.2 (1989): 317-323.

14. Yang JK., et al. "Binding of SARS Coronavirus to its Receptor Damages Islets and Causes Acute Diabetes". Acta Diabetologica 47 (2010): 193-199.

15. Luzi L and Radaelli. "Influenza and Obesity: its Odd Relationship and the Lessons for COVID-19 Pandemic". Acta Diabetologica 57 (2020): 759-764.

16. Nie W., et al. "Obesity Survival Paradox in Pneumonia: A Meta -Analysis". BMC Medicine (2014).

17. Kahlon S., et al. "Obesity and Outcomes in Patients Hospitalized with Pneumonia”. Infectious Diseases 19 (2013): 709-716.

18. Ye Q., et al. "Cytokine storm in COVID-19 and Treatment". Journal of Infection 80.6 (2020): 607-613.

19. Carella MA., et al. "Pharmacological Approach and Therapeutic Options for SARs-CoV-2 Infection”. World Journal of Advanced Research and Reviews (2020). 
20. Subedi S., et al. "Infliximab and Biosimilar Infliximab in Psoriasis: Efficacy, Loss of Efficacy and Adverse Events". Drug Design, Development and Therapy 13 (2019): 2491-2502.

21. Hamming I., et al. "Tissue Distribution of ACE2 Protein, the Functional Receptor for SARS Coronavirus. A first step in understanding SARS Pathogenesis". Journal of Pathology 203 (2004): 631-637.

22. Shereen MA., et al. "COVID-19 Infection: Origin, Transmission, and Characteristics of Human Corona Virus". Journal of Advanced Research 24 (2020): 91-98.

23. Yao H., et al. "Patient - derived Mutations Impact Pathogenicity of SARs-CoV-2". medRxiv (2020).

24. Min J and Jang YJ. "Macrolide Therapy in Respiratory Viral Infections". Mediators of Inflammation (2012).

25. Schogler A., et al. "Novel Antiviral Properties of Azithromycin in Cystic Fibrosis Airway Epithelial Cells". Cystic Fibrosis (2014).

26. Gielen V., et al. "Azithromycin Induces Anti-Viral Responses in Bronchial Epithelial Cells". European Respiratory Journal 36 (2010): 646-654.

27. Blazek K., et al. "IFN- $\lambda$ Resolves Inflammation via Suppression of Neutrophil Infiltration and IL1- $\beta$ Production". The Journal of Experimental Medicine 212.6 (2015): 845-853.

28. Xue J., et al. "Chloroquine is a Zinc Ionophore”. PLOS one (2014).

29. Wang QL., et al. “Ginsenoside Rg1 Regulates SIRT1 to Reduce Sepsis Induced Lung Inflammation and Injury Via Inhibiting Endoplasmic Reticulum Stress and Inflammation". Mediators of Inflammation (2019).

30. Gao J., et al. "Breakthrough: Chloroquine Phosphate has Shown Apparent Efficacy in Treatment of COVID-19 Associated Pneumonia in Clinical Studies". BioScience Trends 14.1 (2020): 7273.

31. Liu J., et al. "Hydroxychloroquine, a Less Toxic Derivative of Chloroquine, is Effective in Inhibiting SARS-COV-2 Infection in Vitro". Cell Discovery (2020).

32. Yao X., et al. "In Vitro Activity and Projection of Optimized Dosing Design of Hydroxychloroquine for the Treatment of Severe Acute Respiratory Syndrome Coronavirus 2 (SARS-CoV-2)". Clinical Infectious Diseases 71.15 (2020): 732-739.
33. Borba M., et al. "Effect of High vs Low Doses of Chloroquine Diphosphate as Adjuvant Therapy for Patients Hospitalized with Severe Acute Respiratory Syndrome Coronavirus-2 (SARSCoV-2) Infection - A Randomized Clinical Trial. Infectious Diseases". JAMA (2020).

34. Smee DF., et al. "Intracellular metabolism of favipiravir (T-705) in uninfected and influenza A (H5N1) virus-infected cells". The Journal of Antimicrobial Chemotherapy (2009).

35. Du YX and Chen XP. "Favipiravir: Pharmacokinetics and Concerns About Clinical Trials for 2019-nCoV Infection". Clinical Pharmacology and Therapeutics (2020).

36. Cai Q., et al. "Experimental Treatment with Favipiravir for COVID-19". Engineering (2020).

37. Shiraki K., et al. "Favipiravir, an Anti-influenza Drug Against Life Threatening RNA Virus Infections". Pharmacology and Therapeutics 209 (2020).

38. Mastrangelo E., et al. "Ivermectin is a Potent Inhibitor of Flavivirus Replication Specifically Targeting NS3 Helicase Activity: New Prospects for and Old Drug". Journal of Antimicrobe Chemotherapy 67 (2020): 1884-1894.

39. Canga AG., et al. "The Pharmacokinetics and Interactions of Ivermectin in Humans - A Mini-review". AAPS Journal (2007).

40. Agostini ML., et al. "Coronavirus Susceptibility to the Antiviral Remdesivir (GS-5734) Is Mediated by the Viral Polymerase and the Proofreading Exoribonuclease". $m B I O$ (2018).

41. Ohmagari JG., et al. "Compassionate Use of Remdesivir for Patients with Severe COVID-19". New England Journal of Medicine (2020).

42. Silverman E., et al. "New Data on Gileads Remdesivir, Released by Accident, Show No Benefit for Coronavirus Patients". STAT (2020).

43. Herod MR., et al. "The Broad -Spectrum Anti-Viral Drug Arbidol Inhibits Foot-and-Mouth Virus Genome Replication". Journal of General Virology 100.9 (2019).

44. Fink SL., et al. "The Anti-Viral Drug Arbidol Inhibits Zika Virus". Scientific Reports (2018).

45. Zhu Z., et al. "Arbidol Monotherapy is Superior to Lopinavir/ Ritonavir in Treating COVID-19". Journal of Infection 31.2 (2020). 
46. Deng L., et al. "Arbidol combined with $\mathrm{LPV} / \mathrm{r}$ versus $\mathrm{LPV} / \mathrm{r}$ alone against Corona Virus Disease 2019: A retrospective cohort study". Journal of Infection (2020).

47. Dong L., et al. "Discovering Drugs to Treat Coronavirus Disease 2019 (COVID-19)". Drug Discoveries and Therapeutics 14.1 (2020): 58-60.

48. Hemlia H. "Zinc Lozenges and the Common Cold: A MetaAnalysis Comparing Zinc Acetate and Zinc Gluconate, and the Role of Zinc Dosage". Journal of the Royal Society of Medicine 8.5 (2017).

49. Skrajnowska D and Bobrowska-Korczak B. "Role of Zinc in Immune System and Anti-Cancer Defense Mechanisms". Nutrients 11 (2019): 2273.

50. Kaushik N., et al. "Zinc Salts Block Hepatitis E Replication by Inhibiting the Activity of Viral RNA-Dependent RNA Polymerase". Journal of Virology (2017).

51. Canna SW., et al. "Making sense of the cytokine storm: a conceptual framework for understanding, diagnosing, and treating hemophagocytic syndrome". Pediatric Clinics of North America (2012).

52. Contassot E., et al. "Interleukin-1, inflammasomes, autoinflammation and the skin". Swiss Medical Weekly (2012).

53. Monteagudo LA., et al. "Continuous Intravenous Anakinra Infusion to Calm the Cytokine Storm in Macrophage Activation Syndrome". ACR Open Rheumatology (2020).

54. Hong D., et al. "Interleukin 1 Inhibition with Ankinra in Adult -Onset Still Disease: A Meta-Analysis of its Efficacy and Safety". Drug Design, Development, and Therapy 8 (2014): 2345-2357.

55. O'Shea JJ., et al. "Back to the Future: Oral Targeted Therapy for RA and Other Autoimmune Diseases". Nature Review Rheumatology 9 (2013): 1713-1782.

56. Alunno A., et al. "Pathogenic and Therapeutic Relevance of JAK/STAT Signaling in Systemic Lupus Erythematosus: Integration of Distinct Inflammatory Pathways and the Prospect of Their Inhibition with an Oral Agent". Cells 8.8 (2019): 898.

57. Choy EHS., et al. "The Effect of JAK1/JAK2 Inhibition in Rheumatoid Arthritis: Efficacy and Safety of Baricitnib". Clinical and Experimental Rheumatology 37 (2019): 694-704.

58. Navarro-Milan I., et al. "Systemic Review of Tocilizumab for Rheumatoid Arthritis: A New Biologic Targeting the Interleukin-6 Receptor". Clinical Therapy 34.4 (2012): 788-802.
59. Sheppard M., et al. "Tocilizumab". Human Vaccines and Immunotherapeutics (2017).

60. Bastard JP., et al. "Evidence for a Link Between Adipose Tissue Interleukin-6 Content and Serum C-Reactive Protein Concentrations in Obese Subjects". Circulation 99 (1999).

61. Genovese MC., et al. "Interleukin-6 receptor inhibition with tocilizumab reduces disease activity in rheumatoid arthritis with inadequate response to disease-modifying antirheumatic drugs: The tocilizumab in combination with traditional disease-modifying antirheumatic drug therapy study". Arthritis and Rheumatism 58.10 (2008): 2968-2980.

62. Emery P., et al. "Safety and tolerability of subcutaneous sarilumab and intravenous tocilizumab in patients with rheumatoid arthritis". Rheumatology 58.5 (2019): 849-858.

63. Korneev KV., et al. "TLR-signaling and proinflammatory cytokines as drivers of tumorigenesis". Cytokine 89 (2017).

64. Wang J., et al. "A Systemic Review on the Efficacy and Safety of Infliximab in Patients with Psoriasis". Human Vaccines and Immunotherapeutics 12.2 (2016).

65. Cheon JA. "Understanding the Complications of Anti-Tumor Necrosis Factor Therapy in East Asian Patients with Inflammatory Bowel Disease". Journal of Gastoenterology and Hepatology 32 (2017): 769-777.

66. Zhao S., et al. "Review of Biosimilar Trials and Data on Adalimumab in Rheumatoid Arthritis". Current Rheumatology Reports 20.10 (2018): 57.

67. Lewandowska K., et al. "The Role of Melatonin and Melatonin Receptor Agonist in the Prevention of Sleep Disturbances and Delirium in Intensive Care Unit - A Clinical Review". Sleep Medicine 69 (2020): 127-134.

68. Lewis SR., et al. "Melatonin for the Promotion of Sleep in Adults in Intensive Care Unit". The Cochrane Data Base of Systematic Review (2018).

69. Foley HM and Steel AE. "Adverse events associated with oral administration of melatonin: A critical systematic review of clinical evidence". Complementary Therapies in Medicine 42 (2018): 65-81.

70. Mehta P., et al. "COVID-19: Consider Cytokine Storm Syndromes and Immunosuppression". Lancet 395 (2020): 10341035. 
71. Russell CD., et al. "Clinical Evidence Does Not Support Corticosteroid Treatment for 2019-nCoV Lung Injury". Lancet 395 (2020): 473-475.

72. Ni Y., et al. "The Effect of Corticoid Steroids on Mortality of Patients with Influenza Pneumonia A Systematic Review and Meta -Analysis". Critical Care 23 (2019): 99.

73. Zhang W., et al. "Anti-inflammation Treatment of Severe Coronavirus Disease 2019 (COVID-19): from the Perspective of Clinical Immunologist”. Clinical Immunology (2019).

74. Weis SM and Cheresh DA. "Pathophysioloigcal Consequences of VEGF-iInduced Vascular Permeability”. Nature 437 (2005): 597-504.

75. Barrat S., et al. "Vascular Endothelial Growth Factor in Acute Lung Injury and Acute Respiratory Distress". Respiration 87 (2014): 329-342.

76. Hamada S., et al. "Protective Effect of Bevacizumab on Chemotherapy -Related Acute Exacerbation of Interstitial Lung Disease in Patients with Advanced Non-Squamous Non-Cell Lung Cancer". BMC Pulmonary Medicine (2019).

77. Semenza GL. "A new weapon for attacking tumor blood vessels". New England Journal of Medicine 358.19 (2008): 20662067.

78. Zhao D., et al. "A Comparative Study on the Clinical Features of COVID-19 Pneumonia to Other Pneumonias". Clinical Infectious Disease 71.15 (2020): 756-761.

79. Zhang JJ., et al. "Clinical Characteristics of 140 Patients Infected with SARS-CoV-2 in Wuhan China”. Allergy (2020).

80. Liu J., et al. "Overlapping and Discrete Aspects of the Pathophysiology and Pathogenesis of the Emerging Human Pathogenic Coronaviruses SARS-CoV, MERS-CoV, and 2019-nCoV". Medical Virology (2020).

\section{Assets from publication with us}

- Prompt Acknowledgement after receiving the article

- Thorough Double blinded peer review

- Rapid Publication

- Issue of Publication Certificate

- High visibility of your Published work

Website: www.actascientific.com/

Submit Article: www.actascientific.com/submission.php

Email us: editor@actascientific.com

Contact us: +919182824667

Citation: Joseph Angel de Soto., et al. "Complementary Pharmacological Treatment and Therapeutic Prospects for COVID-19". Acta Scientific Pharmaceutical Sciences 4.11 (2020): 82-89. 